\title{
Enzymatic hydrolysis of orange bagasse and effect of filtration on lactic acid fermentation
}

\author{
Saadet Fatma Baltaci ${ }^{1,2}\left(\right.$ D $\cdot$ Haluk Hamamci $^{1,2}$
}

Received: 14 November 2019 / Accepted: 4 March 2020 / Published online: 11 March 2020

(c) Springer Nature Switzerland AG 2020

\begin{abstract}
Along with the general trend of reducing waste in the agro-industrial sector, new methods are sought to utilize side or waste products. With this purpose, we examined the utilization of orange bagasse (OB) for Ca-lactate production via enzymatic hydrolysis and fermentation. The hydrolysis of $\mathrm{OB}$ was carried in both dried and fresh forms with cellulolytic and pectinolytic enzymes to obtain a reasonable amount of fermentable sugars for further lactic acid fermentation. A novel industrial approach was studied to increase the hydrolysis yield by reusing the former hydrolysate media. Meanwhile, lactic acid fermentation was performed with optimal OB hydrolysate by Lactobacillus delbrueckii subsp. bulgaricus $\mathrm{OZZ4}$ and Lactobacillus plantarum OZH8. One of the main problems in OB utilization via fermentation was the inhibition observed on microorganisms. It was found that either by drying of the biomass prior to enzymatic hydrolysis or by filtration of the hydrolysate prepared with fresh biomass could be done in order to overcome the inhibitory effects. After treatments, lactic acid with yields around $0.95 \mathrm{~g} / \mathrm{g}$ and levels of $45 \mathrm{~g} / \mathrm{L}$ was produced with the fermentation of OB hydrolysate.
\end{abstract}

Keywords Orange bagasse $\cdot$ Enzymatic hydrolysis $\cdot$ Lactic acid $\cdot$ Fermentation $\cdot$ Filtration

\section{Introduction}

In recent years, there has been an increasing tendency in the utilization of agricultural or organic industrial wastes such as straws, cobs, paper and wood pulps, residues of crops or processed feedstocks for the production of green and degradable biopolymers, and bioenergy as a substitute for petroleum-based raw materials and energy sources [1]. Hence, the bioconversion of several organic substances such as a citrus waste has gained importance due to the rising prices of raw materials as starch, sucrose, etc.

Orange bagasse $(\mathrm{OB})$ comprises the significant part of citrus waste and can be converted into several bio-products thanks to its lignocellulosic structure and pectin content. According to FAO Citrus Fruit Statistics published in 2016, 68.9 MT of orange were produced around the world and 21.2 MT of orange were utilized for processing, which constituted almost $80 \%$ of total utilized citrus fruits [2]. A great majority of orange and other citrus fruits are used in fruit juice industry and approximately $50 \%$ of total weight of the fruits are disposed as industrial waste, which poses economic and environmental problems even though some parts of them are utilized as animal feed [3].

Orange bagasse is mainly composed of peel, inner membrane, seeds and pulp having various soluble and non-soluble carbon sources, essential oils, etc. which make

Electronic supplementary material The online version of this article (https://doi.org/10.1007/s42452-020-2421-0) contains supplementary material, which is available to authorized users.

Saadet Fatma Baltaci, saadetbaltaci@gmail.com; baltaci.saadet@metu.edu.tr; Haluk Hamamci, hhamamci@metu.edu.tr| ${ }^{1}$ Department of Food Engineering, Middle East Technical University, Ankara, Turkey. ${ }^{2}$ H2Biyotek Co., METU Technopolis, MET Area, Cankaya, Ankara, Turkey. 
it a valuable raw material for the production of ethanol, organic acids, pectin and limonene. By enzymatic saccharification of holocellulose and pectic compounds constituting approximately $70 \%$ of OB in dry weight, simple sugars which can be used in the fermentation process are obtained [4]. However, the efficiency of these processes can be negatively affected by product inhibition or the existence of antimicrobial compounds in the biomass. Hence, the fermentation of OB hydrolysate for bioethanol or organic acid production is generally preceded by pretreatment steps. In many studies, OB is subjected to various physical, chemical or physicochemical pretreatments to remove limonene, which possesses antimicrobial activity [5]. However, these methods, especially chemical ones could increase the energy input and the cost of the process.

One of the products that can be produced by bioprocessing of orange waste is lactic acid or 2-hydroxypropanoic acid, which is widely used in several industries such as food, pharmaceutical, chemical, etc. with various purposes. Approximately $90 \%$ of lactic acid is produced by fermentation to obtain optically pure and heat stable isomers of lactic acid in contrary to chemical methods by which racemic DL-lactic acid is produced $[6,7]$. Recently the production of poly-lactic acid (PLA) polymers from optically pure isomers has gained importance due to the biodegradable and eco-friendly properties of PLA-based plastics, as a substitute for the petroleum-based ones [8].

In the present study, we investigated the enzymatic saccharification of orange bagasse with cellulolytic and pectinolytic enzymes and the effect of filtration of hydrolysate as preparation steps for the lactic acid fermentation by two Lactobacillus species.

\section{Material and methods}

\subsection{Pretreatment of orange bagasse}

Orange bagasse was collected from local orange juice producers; Belso A.Ş. and Susam Bakery in Ankara, Turkey and stored at $-20^{\circ} \mathrm{C}$ in deep freezer. Before the enzymatic hydrolysis and lactic acid fermentation, only physical pretreatment methods were applied to OB. Prior to hydrolysis, the particle size of fresh orange bagasse (FOB) was reduced to $1-2 \mathrm{~mm}$ by using a bench-top mincing machine. FOB was dried in an oven incubator at $80^{\circ} \mathrm{C}$ until the moisture content of $\mathrm{OB}$ decreased below $10 \%$ and dried orange bagasse (DOB) was milled to $1 \mathrm{~mm}$ homogenous particle size (Laboratory Mill, Philadelphia, USA). The moisture content of $O B$ was determined by using infrared moisture analyzer (Radwag MAC 50, Poland).

\subsection{Enzymatic hydrolysis of orange bagasse}

In order to obtain fermentable sugars, pretreated $O B$ was hydrolyzed with the celluloytic and pectinolytic enzymes, which are ASA Biogazyme 2x (ASA-BG, a mixture of exo-cellulase (EC 3.2.1.91), endo-glucanase (EC 3.2.1.4), $\beta$-glucosidase (EC 3.2.1.21), xylanase (EC 3.2.1.8), endo-mannanase (EC 3.2.1.78), $\beta$-1,3(4)-glucanase (EC 3.2.1.73) and ASA Pektinase L40 (ASA-P, a mixture of exo, endo- polygalacturonase and pectindepolymerase (EC.3.2.1.15)), respectively. The saccharification of $O B$ was performed with $100 \mathrm{~mL}$ hydrolysis medium, which contained $0.05 \mathrm{M}$ sodium-citrate buffer at $4.8 \mathrm{pH}$, orange bagasse and enzymes at different concentrations, in $250 \mathrm{~mL}$ Erlenmeyer flasks in shaking incubators (Infors HT, Switzerland). The hydrolysis was conducted at $55^{\circ} \mathrm{C}, 150 \mathrm{rpm}$ and the $\mathrm{pH}$ of the hydrolysis medium was adjusted to 4.8 following the first hour of the experiment with the use of $2 \mathrm{M} \mathrm{NaOH}$.

\subsubsection{Effect of substrate and enzyme loading}

The effect of solid load was examined by hydrolyzing $2 \%, 5 \%, 10 \%$ and $20 \%(\mathrm{w} / \mathrm{v})$ of DOB on dry basis with $130 \mathrm{FPU}$ of ASA-BG and $2320 \mathrm{IU}$ of ASA-P. To observe the effect of enzyme loading, DOB ( $10 \% \mathrm{w} / \mathrm{v}$ solid load in dry weight) was hydrolyzed by ASA-BG and ASA-P enzymes in different amounts changing between 0.13 FPU and $65 \mathrm{FPU}$ per gram OB, and $2.3 \mathrm{IU}$ and $1160 \mathrm{IU}$ per gram $\mathrm{OB}$, respectively.

\subsubsection{Recycle of hydrolysis medium}

Hydrolysis process was repeated for three times to increase the sugar concentration, which leads to higher lactic acid titer. The enzymatic hydrolysis was done by using 10\% DOB with 13 FPU ASA-BG and 232 IU ASA-P per gram biomass for $24 \mathrm{~h}$. At the end of each step, hydrolysis medium was centrifuged at $14,000 \mathrm{~g}$ for $10 \mathrm{~min}$ and the supernatant was used as a buffer of the next stage. In the beginning of each stage, only the dried biomass $(10 \% \mathrm{w} / \mathrm{v})$ was added to the medium.

\subsection{Filtration of orange bagasse hydrolysate}

In order to observe the effect of filtration on lactic acid fermentation, a second set of biomass treatment was done. In this step, the DOB and FOB hydrolysates obtained after the first hydrolysis process were centrifuged at $14,000 \mathrm{~g}$ for $10 \mathrm{~min}$ (Sigma Laborzentrifugen $\mathrm{GmbH}$, Germany) and then centrifugated FOB 
hydrolysate was filtered through 0.45 micron filter in a lab-scale vacuum filtration apparatus. The aim of the centrifugation was to aid the filtration step.

\subsection{Lactic acid fermentation}

\subsubsection{Microorganisms and batch fermentation}

Strains of lactic acid bacteria (LAB); Lactobacillus delbrueckii subsp. bulgaricus OZZ4 and Lactobacillus plantarum $\mathrm{OZH} 8$ were kindly provided by Dr. Özlem Osmanağaoğlu and her group in Ankara University in Turkey. The bacteria were stored at $-80^{\circ} \mathrm{C}$ in a $1: 1$ ratio of liquid growth medium and $50 \%$ glycerol solution as stock culture. Inoculum was prepared by cultivating strains from the stock culture into sterilized $5 \mathrm{~mL}$ test tubes containing MRS broth (Merck, Germany), and incubated at $38^{\circ} \mathrm{C}$ and $160 \mathrm{rpm}$ for $14 \mathrm{~h}$ to obtain an activated culture.

The activated main culture $(5 \mathrm{~mL})$ was inoculated into a $250 \mathrm{~mL}$ conical flask with $95 \mathrm{~mL}$ fermentation medium. The medium which was sterilized at $121^{\circ} \mathrm{C}$ for 15 min before inoculation contained fresh or dried OB hydrolysate, enhanced MRS broth ( $10 \mathrm{~g} / \mathrm{L}$ peptone, $20 \mathrm{~g} / \mathrm{L}$ yeast extract, $2 \mathrm{~g} / \mathrm{L}$ dipotassium hydrogen phosphate, $5 \mathrm{~g} / \mathrm{L}$ sodium acetate trihydrate, $2 \mathrm{~g} / \mathrm{L}$ triammonium citrate, $1 \mathrm{~g} / \mathrm{L}$ Tween, $0.2 \mathrm{~g} / \mathrm{L}$ magnesium sulfate heptahydrate and $0.05 \mathrm{~g} / \mathrm{L}$ manganous sulfate tetrahydrate) as $2 \%$ of total process volume and $30 \mathrm{~g} / \mathrm{L} \mathrm{CaCO}_{3}$ as a neutralizer. Lactic acid fermentation was conducted at $38^{\circ} \mathrm{C}, 160 \mathrm{rpm}$ in shaking incubators (Infors HT, Switzerland) with the general ratio of orange bagasse in hydrolysate and eMRS broth as 5:1.

\subsection{Sample analysis}

The samples of hydrolysis experiments were taken at various time intervals, treated by heat at $100^{\circ} \mathrm{C}$ for $10 \mathrm{~min}$ to deactivate the enzymes and centrifuged at $18,180 \mathrm{~g}$ for $5 \mathrm{~min}$. The supernatant of each sample was diluted properly, filtered through $0.22 \mu \mathrm{m}$ nylon filters and analyzed with HPLC (Agilent Technologies, USA) equipped with Rezex RPM-Monosaccharide column $(300 \times 7.8 \mathrm{~mm})$ at $85^{\circ} \mathrm{C}$ and a refractive index detector at $55^{\circ} \mathrm{C}, 10 \mu \mathrm{L} \mathrm{sam}$ ple injection and $0.6 \mathrm{~mL} / \mathrm{min}$ flow rate of double distilled water.

In order to determine the concentration of 5- and 6-carbon sugars and lactic acid in the fermentation medium, aseptically taken samples were centrifuged with the same settings given above. Then, the supernatants of samples were diluted, filtered $(0.22 \mu \mathrm{m}$ nylon filter) and analyzed with HPLC (Agilent Technologies, USA) equipped with Rezex ${ }^{\mathrm{TM}} \mathrm{RFQ}$-Fast Acid $\mathrm{H}+(8 \%) \mathrm{LC}$ column at $25^{\circ} \mathrm{C}$ and refractive index detector at $30^{\circ} \mathrm{C}$. As an eluent, $0.05 \mathrm{M}$ $\mathrm{H}_{2} \mathrm{SO}_{4}$ solution was used at $0.6 \mathrm{~mL} / \mathrm{min}$ flow rate.

\subsection{Data analysis}

The mean values of the results of replicated experiments were presented with the standard error data. The experiment results were statistically analyzed with the analysis of variance (ANOVA) by using the software Minitab 16.2.0.0 (Minitab Inc., UK). The Tukey Test with 95\% confidence level was applied for the comparison of data.

\section{Results and discussion}

\subsection{Enzymatic saccharification of orange bagasse}

To investigate the effect of enzyme loading on saccharification of orange bagasse, the hydrolysis experiments were conducted with a wide range of enzyme dosage. Figure 1 represents the sugar conversion data obtained at the end of 24 th hour of hydrolysis conducted with DOB $(10 \%$ solid load). The enzymatic hydrolysis were performed with low and high dose of enzyme mixture; $0.13 \mathrm{FPU}-65 \mathrm{FPU}$ of ASA-BG and 2.3 IU-1160 IU of ASA-P per gram biomass. It should be noted that, the sugar conversion data at the 24th hour of hydrolysis were graphed and the total sugar data of hydrolysis experiments were given as the sum of glucose, xylose, galactose and arabinose concentrations.

As shown in Fig. 1, the sugar conversion proportionally increased with the increase in enzyme amount up to $2.6 \mathrm{FPU} / \mathrm{g}$ and $46 \mathrm{IU} / \mathrm{g}$. Further increases in the enzyme load also resulted in significant changes in sugar conversion even though they were not proportional to each other. Meanwhile, the hydrolysis experiments were performed with high dose of enzyme to increase the fermentable sugar concentration. As can be seen, the glucose conversion significantly but not proportionally changed at the end of hydrolysis by increasing the enzyme concentrations at high dosage. On the other hand, the significant change was observed in total sugar conversion after the use of $13 \mathrm{FPU} / \mathrm{g}$ and $232 \mathrm{lU} / \mathrm{g}$ of cellulase and pectinase mixture. In addition, when the amount of enzyme increased five-fold from sample 6 to 10 , the glucose conversion increased from 12.1 to $23.1 \mathrm{~g} / \mathrm{L}$ and the total sugar conversion only increased from 19.3 to $31.4 \mathrm{~g} / \mathrm{L}$. This situation could be explained by different phenomena reported in previous studies such as product inhibition or the effect of limiting factors in adsorption of cellulose [9, 10]. According to Kristensen et al., an increase in the end-product concentration could decrease the conversion efficiency [9]. They reported that an increase in glucose concentration inhibited the activity of $\beta$-glucosidase. Another factor was explained by Mansfield et al. [10] that the adsorption of 


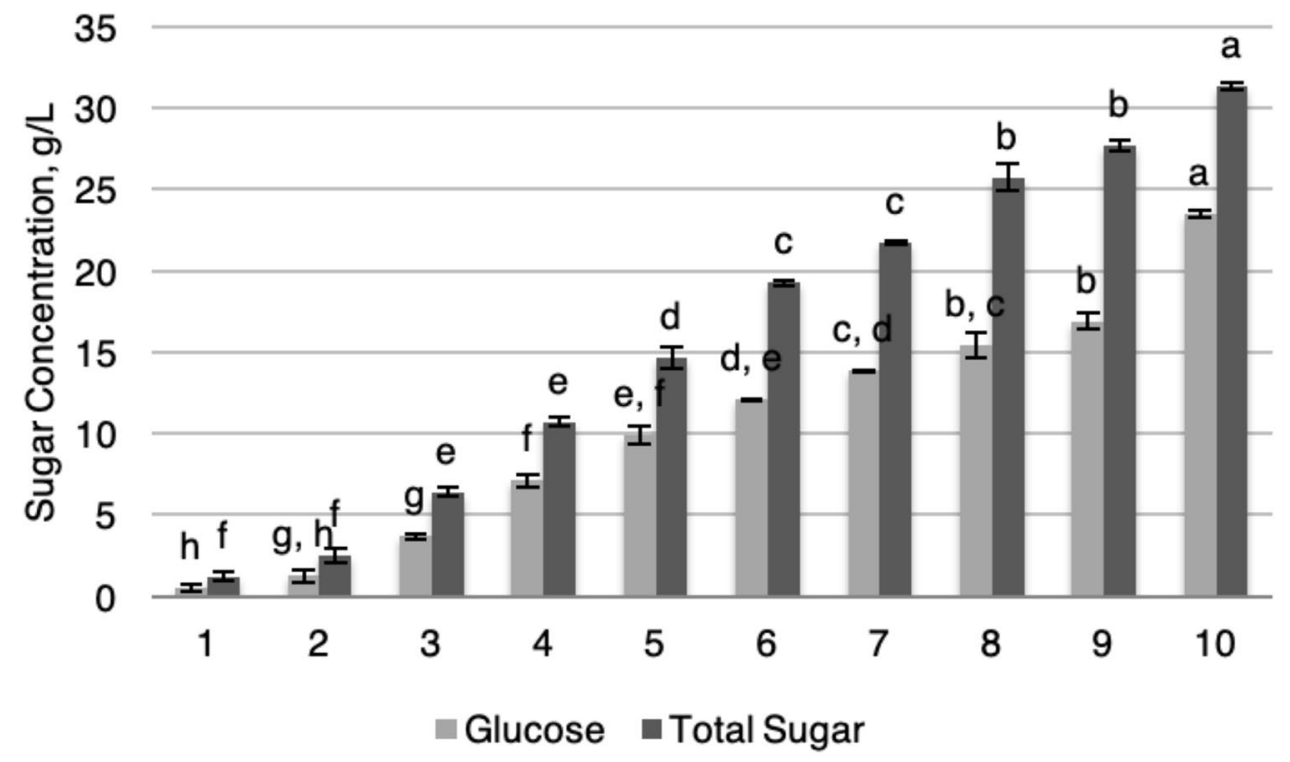

Fig. 1 The effect of low and high dose of enzyme mixture on sugar conversion in $\mathrm{g} / \mathrm{L}$ by the hydrolysis with $10 \%(\mathrm{w} / \mathrm{v})$ of DOB (1: 0.13 FPU ASA-BG and 2.3 IU ASA-P, 2: 0.65 FPU ASA-BG and 12 IU ASAP, 3: 1.3 FPU ASA-BG and 23 IU ASA-P, 4: 2.6 FPU ASA-BG and 46 IU ASA-P, 5: 6.5 FPU ASA-BG and 116 IU ASA-P; 6: 13 FPU ASA-BG and

enzyme onto cellulose could be diversely affected from the degree of crystallinity of treated biomass; as a result, the efficiency of hydrolysis could decrease.

Besides, the hydrolysis experiments with high dosage enzyme mixture continued up to $96 \mathrm{~h}$ by analyzing daily taken samples; however, more than $80 \%$ conversion occurred at the end of 24th hour. Nevertheless, it should be mentioned that the continuation of hydrolysis for $96 \mathrm{~h}$ caused approximately $10-15 \mathrm{~g} / \mathrm{L}$ increase in total sugar

232 IU ASA-P, 7: 26 FPU ASA-BG and 464 IU ASA-P, 8: 39 FPU ASA-BG and 696 IU ASA-P, 9: 52 FPU ASA-BG and 928 IU ASA-P, 10: 65 FPU ASA-BG and 1160 IU ASA-P per gram OB) The significant difference was represented by different letters $(p \leq 0.05)$ and one-way ANOVA was applied separately for glucose and total sugar conversion

conversion, which could be a considerable amount in industrial scale processing (for data see Online Source 1).

Another important criterion for high process efficiency is the amount of biomass to be hydrolyzed. Figure 2 illustrates the effect of solid load on sugar conversion, which increased gradually up to $10 \%(\mathrm{w} / \mathrm{v})$ of the substrate load. However, no significant difference on sugar concentration was obtained when the solid load of OB increased from 10 to $20 \%(p \leq 0.05)$. At $10 \%$ solid load, $12.1 \mathrm{~g} / \mathrm{L}$ glucose and
Fig. 2 Effect of substrate loading on glucose and total sugar conversion in $\mathrm{g} / \mathrm{L}$ by the hydrolysis with $13 \mathrm{FPU} / \mathrm{g}$ ASA-BG and 232 IU ASA-P (1: $2 \%$ solid load, $2: 5 \%$ solid load, 3: $10 \%$ solid load, 4: $20 \%$ solid load). The significant difference was represented by different letters $(p \leq 0.05)$ and one-way ANOVA was applied separately for glucose and total sugar conversion data

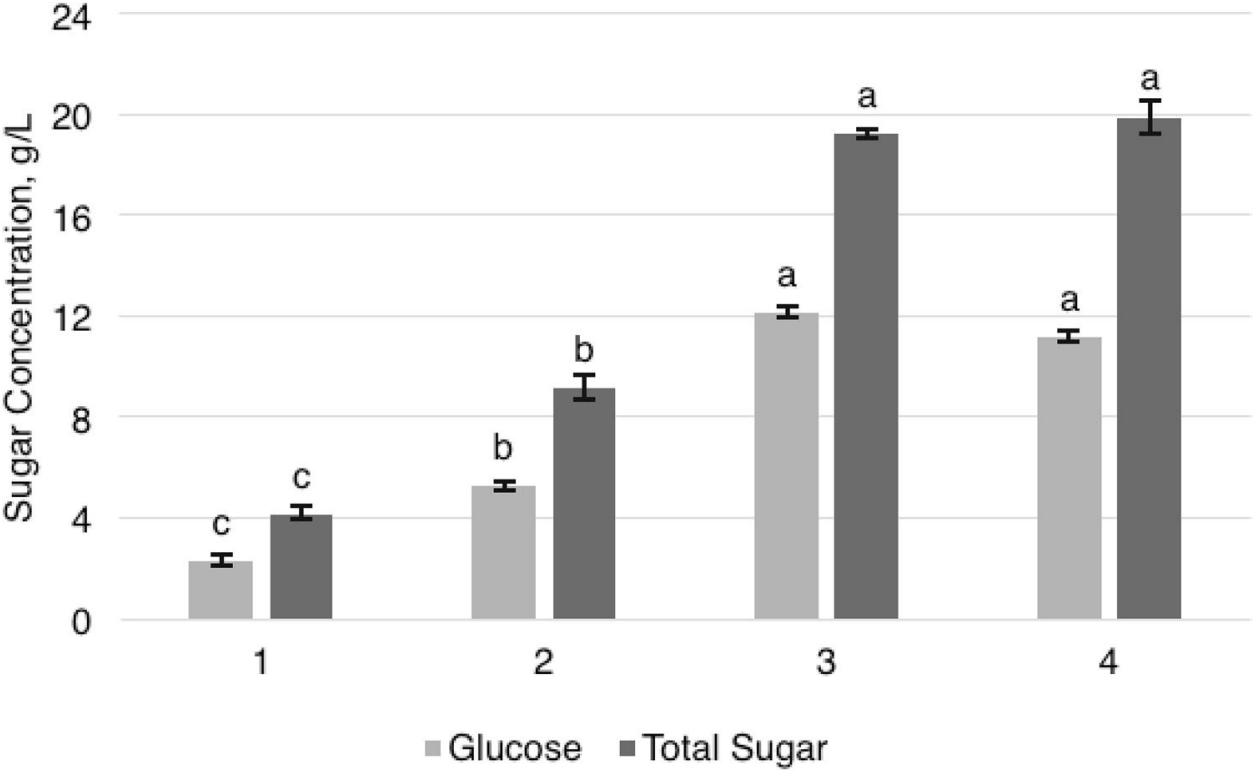


$19.3 \mathrm{~g} / \mathrm{L}$ total sugar converted while $11.2 \mathrm{~g} / \mathrm{L}$ glucose and $19.9 \mathrm{~g} / \mathrm{L}$ total sugar converted at $20 \%$ solid load. These results could also be supported by the study of Kristensen et al., in which the yield decreased due to the inhibition on cellulase adsorption was reported. They showed that the inhibition might occur because the high solid concentration, which resulted from increased sugar conversion, adversely affected sugar binding domains of the enzyme. However, it should be noted that the reason behind the adsorption inhibition is still not known [11].

In order to increase sugar concentration through enzymatic conversion as well as the lactic acid titer in the fermentation step, recycling of hydrolysis experiments were performed. In this experimental setup, an industrial approach was applied to obtain higher values in fermentable sugar concentration by using a single batch of hydrolysis medium to minimize the water, enzyme, chemicals and energy consumption. According to the Fig. 3 , an increase in the number of recycling steps increased the glucose and total sugar conversion with a lower rate, which could be explained as the loss enzyme activity through the processes. At the end of the third step, the glucose and total sugar conversion almost doubled and reached to $23.4 \mathrm{~g} / \mathrm{L}$ and $34.5 \mathrm{~g} / \mathrm{L}$, respectively. It was seen that the reuse of hydrolysates significantly affected the amount of converted glucose and total sugar concentration.

\subsection{Production of Ca-lactate from orange bagasse}

Figure 4 represents the change in the concentration of glucose and lactic acid during the batch fermentation of $D O B$ hydrolysate ( $10 \%$ solid load) by L. delbrueckii subsp. bulgaricus OZZ4 and L. plantarum OZH8. At the end of fermentation, approximately $46 \mathrm{~g} / \mathrm{L}$ lactic acid was produced by these two strains even though the productivities obtained up to 24th hour of fermentation differed for both of the strains.

The amount of other monomeric sugars consumed by the microorganisms can be seen in Table 1. Both of the microorganisms consumed almost all of the monomeric sugars except xylose. However, any genetic analysis on sugar consumption metabolism of the strains were not performed for the present study to mention in detail why xylose was not consumed due to being out of scope, and any other works using these strains could not be stated because they were genetically modified and kindly provided by Dr. Özlem Osmanağaoğlu and her group in Ankara University to produce D-lactic acid within the scope of this project.

In addition, when lactic acid fermentation was statistically analyzed with a general linear model (GLM), it was observed that the lactic acid concentration obtained at the 6th and 12th hour of fermentation was significantly different between two strains of Lactobacillus. On the other hand, no significant difference was observed on the concentration of lactic acid produced by both of the strains after 24th hour of fermentation. When the total sugar consumption was considered, both microorganisms produced Ca-lactate with high yields $\left(\mathrm{Y}_{\mathrm{L} / \mathrm{T}}\right)$ which were $0.96 \mathrm{~g} / \mathrm{g}$ and $0.95 \mathrm{~g} / \mathrm{g}$, respectively, and no significant difference was also observed $(p \leq 0.05)$.

\subsection{Effect of filtration}

In the literature, various pretreatment methods for citrus wastes can be found. These methods aim an increase in the efficiency of enzymatic hydrolysis by disrupting the lignocellulose structure and increasing surface area of
Fig. 3 Converted glucose and total sugar concentration $(\mathrm{g} / \mathrm{L})$ by repeating hydrolysis with $10 \%(w / v)$ of DOB, and 13 FPU ASA-BG and 232 IU ASA-P per gram biomass. The significant difference was represented by different letters $(p \leq 0.05)$ and one-way ANOVA was applied separately for glucose and total sugar conversion data

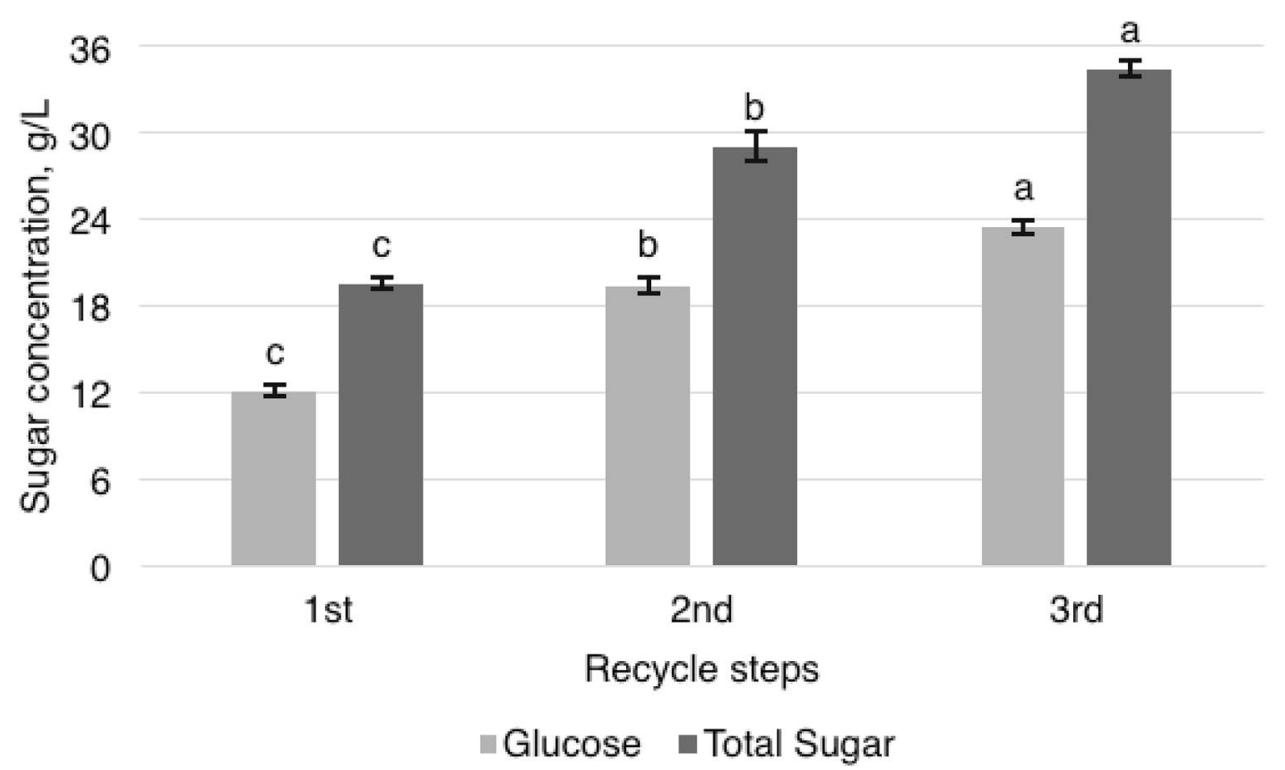

SN Applied Sciences A SPRINGER NATURE journal 
Fig. 4 Glucose and lactic acid concentration in lactic acid fermentation with $10 \%(\mathrm{w} / \mathrm{v})$ of DOB hydrolysate and $5 \%$ inoculum (G: glucose, LA: lactic acid, Id: L. delbrueckii subsp. bulgaricus OZZ4, Ip: L. plantarum $\mathrm{OZH} 8$ )
Table 1 The data of sugar concentration as $\mathrm{g} / \mathrm{L}$ in the medium before and after fermentation (Id: L. delbrueckii subsp. bulgaricus OZZ4, Ip: L. plantarum $\mathrm{OZH} 8$ )
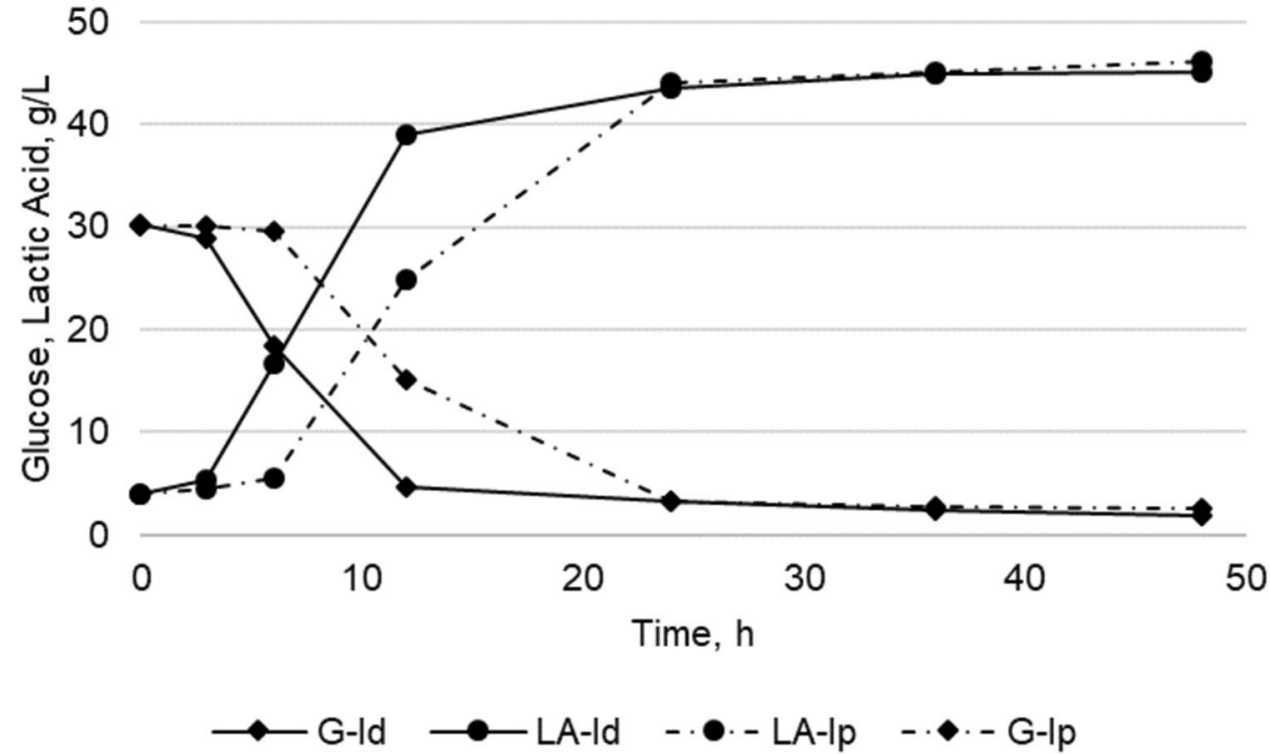

\begin{tabular}{lrlllr}
\hline $\mathrm{LAB}$ & Glucose, $\mathrm{g} / \mathrm{L}$ & Xylose, $\mathrm{g} / \mathrm{L}$ & Galactose, $\mathrm{g} / \mathrm{L}$ & Arabinose, $\mathrm{g} / \mathrm{L}$ & Fructose, $\mathrm{g} / \mathrm{L}$ \\
\hline $\mathrm{Id}_{\text {before }}$ & $30.16 \pm 0.12$ & $1.72 \pm 0.02$ & $4.68 \pm 0.10$ & $3.77 \pm 0.15$ & $13.14 \pm 0.04$ \\
Ip $_{\text {before }}$ & $30.39 \pm 0.39$ & $1.90 \pm 0.03$ & $4.61 \pm 0.11$ & $4.17 \pm 0.10$ & $13.62 \pm 0.16$ \\
$\mathrm{Id}_{\text {after }}$ & $1.13 \pm 0.06$ & $1.40 \pm 0.08$ & $2.00 \pm 0.07$ & $1.39 \pm 0.55$ & $0.84 \pm 0.01$ \\
$\mathrm{IP}_{\text {after }}$ & $1.32 \pm 0.14$ & $1.34 \pm 0.01$ & $1.12 \pm 0.15$ & $1.63 \pm 0.02$ & $2.51 \pm 0.11$ \\
\hline
\end{tabular}

the biomass as well as the removal D-limonene, which is an organic substance, a monoterpene, and constitutes approximately $95 \%$ of the essential oils of orange peel and has an inhibitory effect on microorganisms, from the waste $[5,12,13]$.

In this study, any pretreatment methods except physical ones were not performed to increase the hydrolysis efficiency because of low lignin concentration of orange bagasse [4]. Biomass was only dried and milled to fine particles to decrease the particle size and crystallinity of cellulosic structure. However, drying is an energy consuming process, which significantly increases the cost of total process although it brings benefit in the storage and handling of orange waste. Besides, when the FOB and DOB $(10 \%$ solid load) hydrolyzed with the same amount of enzyme (13 FPU/g ASA-BG and 232/g IU ASA-P), any significant difference in the glucose and total sugar conversion was not observed (for data see Online Source 2). In order to lower the cost of process, fermentation experiments were tried with fresh orange bagasse hydrolysate. Figure 5 illustrates the results of fermentation experiments done with two different media.

The first medium consisted of fresh orange bagasse hydrolysate which was only centrifuged after enzymatic hydrolysis (same procedure applied to DOB) while the second one contained FOB hydrolysate which was both centrifuged and filtered to see the effect of filtration on lactic acid fermentation. As clearly be seen, both of the Lactobacillus strains could not produce lactic acid in the first medium. On the other hand, in the second medium, they converted the fermentable sugars to lactic acid with a remarkably high yield $\left(\mathrm{Y}_{\mathrm{L} / \mathrm{T}}\right)$, which was around $0.95 \mathrm{~g} / \mathrm{g}$ and similar to the ones obtained from the fermentation of DOB hydrolysate which was only centrifuged. The effect of filtration on lactic acid production was statistically examined by GLM and it was proved that the lactic acid concentration was significantly different between two fermentation media except the beginning of the fermentation (the 0th hour of process) for both of the strains.

These results showed that the adverse effects of inhibitory compounds could be eliminated by filtering the fresh orange bagasse hydrolysate after centrifugation as well as by drying and centrifugation. The attachment of inhibitory components to insoluble residues during the hydrolysis could provide their separation from the medium by a simple filtration technique as reported in a previous study of Grohmann et al. They showed that filtration decreased limonene concentration in the filtrate below the inhibition level $(0.05 \%$ and $0.1 \%)$ and allowed them to produce ethanol from orange peel by the yeast S. cerevisiae [14]. The interpretation of the current result could also be strengthened by comparing the inhibition levels of various 
Fig. 5 Effect of filtration on lactic acid fermentation by (a) L. delbrueckii subsp. bulgaricus OZZ4, (b) L. plantarum OZH8 (1: lactic acid fermentation with centrifuged and filtered FOB hydrolysate, 2: lactic acid fermentation with only centrifuged FOB hydrolysate)
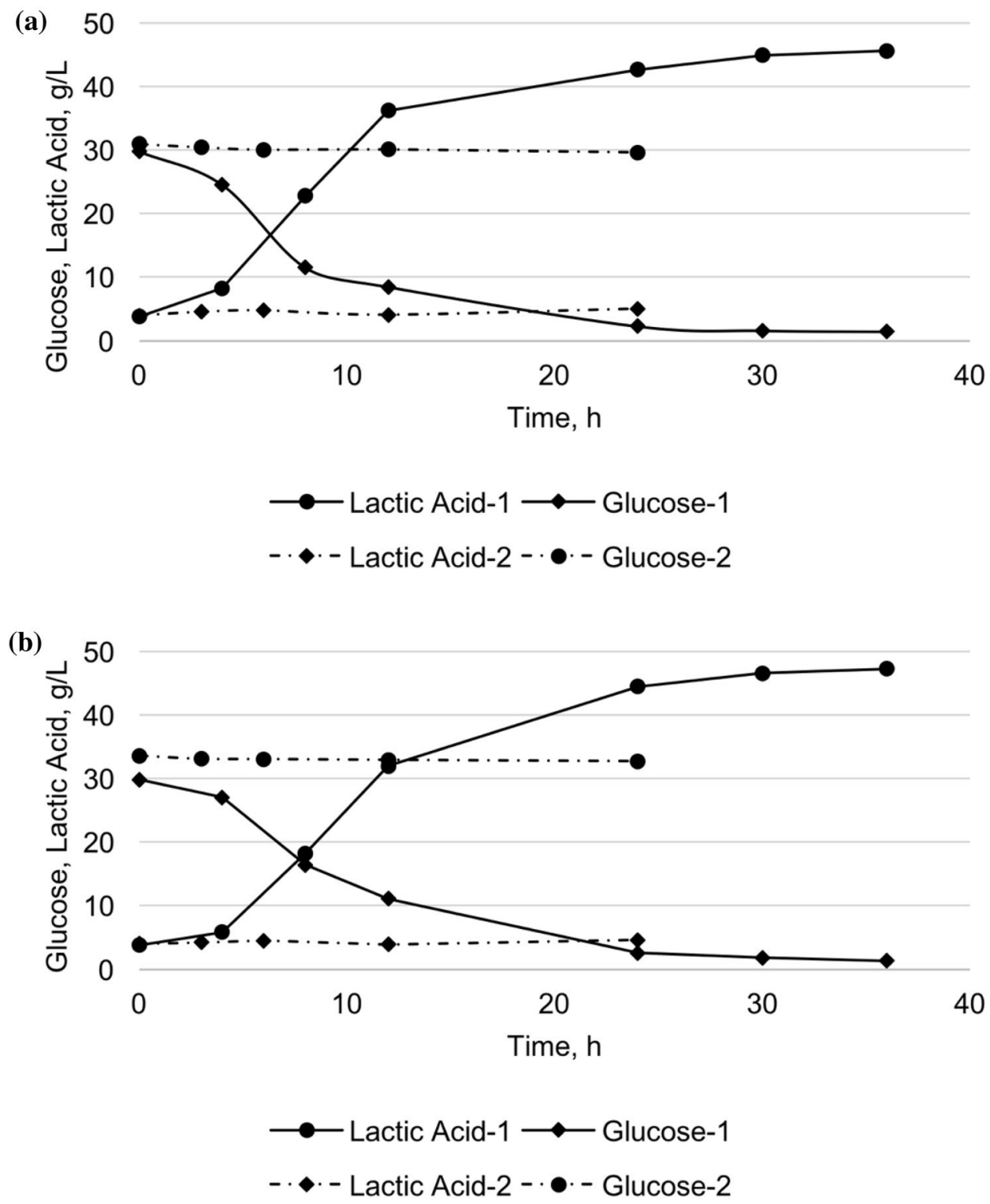

microorganisms with an extensive literature review. For instance, M.R. Zahi et al. reported the minimum concentrations for some strain of E. coli and S. cerevisiae as $1.0 \mu \mathrm{g} / \mathrm{mL}$ and $0.5 \mu \mathrm{g} / \mathrm{mL}$. In another study, Ambrosio et al. stated the minimum levels for the resistant strain of E.coli and sensitive species of Lactobacillus (L. rhamnosus) as $1.85 \mathrm{mg} / \mathrm{mL}$ and $3.70 \mathrm{mg} / \mathrm{mL}$, respectively $[15,16]$.

Furthermore, this study showed that the heat treatment at higher temperatures could help for the elimination of some inhibitory compounds found in orange waste even though the ineffectiveness of vacuum evaporation, a process performed at a lower temperature, on the removal of limonene due to its low volatility pointed out in [14]. As shown, after drying the orange bagasse at $80^{\circ} \mathrm{C}$ overnight, the filtration step was not required as for the fresh biomass.

\section{Conclusion}

The present study covered the saccharification of orange bagasse by cellulase and pectinase, and the lactic acid fermentation by two Lactobacillus strains in the medium containing orange bagasse hydrolysate. It was seen that the hydrolysis of orange bagasse with $10 \%$ solid load $(\mathrm{w} / \mathrm{v})$ and moderate amount of enzyme resulted in the optimal sugar conversion. The inhibitory effects of limonene, an important obstacle in the biorefining of citrus waste, was found to be eliminated by a filtration technique for the fermentation step following the hydrolysis process. The study showed that a simple filtration step was sufficient to prepare orange bagasse hydrolysate for the ensuing fermentation step in which around $46 \mathrm{~g} / \mathrm{L}$ lactic acid was obtained. 
Acknowledgement This study was supported by The Scientific and Technological Research Council of Turkey, TUBITAK (9120050) and conducted in the scope of FP7 ERA-NET project named as "Production of Organic Acids for Polyester Synthesis".

Author contributions Prof. Dr. Haluk Hamamci advised the study and commented on the manuscript. Saadet Fatma Baltaci conducted all the material preparation, experiments, analysis, data evaluation and the manuscript writing. All authors read and approved the final manuscript.

\section{Compliance with ethical standards}

Conflict of interest The authors declare no conflict of interest.

\section{References}

1. Sun Y, Cheng J (2002) Hydrolysis of lignocellulosic materials for ethanol production: a review. Bioresour Technol 83:1-11. https ://doi.org/10.1016/S0960-8524(010)00212-7

2. FAO: citrus fruits statistics 2015 (2016). https://www.fao.org/3/ai5558e.pdf

3. Lohrasbi M, Pourbafrani M, Niklasson C, Taherzadeh MJ (2010) Process design and economic analysis of a citrus waste biorefinery with biofuels and limonene as products. Bioresour Technol 101:7382-7388. https://doi.org/10.1016/j.biortech.2010.04.078

4. Boluda-Aguilar M, López-Gómez A (2013) Production of bioethanol by fermentation of lemon (Citrus limon L.) peel wastes pretreated with steam explosion. Ind Crops Prod 41:188-197. https ://doi.org/10.1016/j.indcrop.2012.04.031

5. Pourbafrani M, Forgács G, Sarvari Horvath I, Niklasson C, Taherzadeh MJ (2010) Production of biofuels, limonene and pectin from citrus wastes. Bioresour Technol 101:4246-4250. https:// doi.org/10.1016/j.biortech.2010.01.077

6. Hofvendahl K, Hahn-Hägerdal B (2000) Factors affecting the fermentative lactic acid production from renewable resources. Enzyme Microb Technol 26:87-107. https://doi.org/10.1016/ S0141-0229(99)00155-6

7. Taniguchi M, Hoshina M, Tanabe S, Higuchi $Y$, Sakai K, Ohtsubo S, Hoshino K, Tanaka T (2005) Production of L-lactic acid by simultaneous saccharification and fermentation using unsterilized defatted rice bran as a carbon source and nutrient components. Food Sci Technol Res 11:400-406. https://doi. org/10.3136/fstr.11.400

8. Taniguchi M, Tokunaga T, Horiuchi K, Hoshino K, Sakai K, Tanaka T (2004) Production of L-lactic acid from a mixture of xylose and glucose by co-cultivation of lactic acid bacteria. Appl Microbiol Biotechnol 66:160-165. https://doi.org/10.1007/s0025 3-004-1671-x

9. Kristensen JB (2008) Enzymatic hydrolysis of lignocellulose: Substrate interactions and high solids loadings. Forest \& Landscape, University of Copenhagen, Copenhagen

10. Mansfield SD, Mooney C, Saddler JN (1999) Substrate and enzyme characteristics that limit cellulose hydrolysis. Biotechnol Prog 15:804-816. https://doi.org/10.1021/bp9900864

11. Kristensen JB, Felby C, Jørgensen H (2009) Yield-determining factors in high-solids enzymatic hydrolysis of lignocellulose. Biotechnol Biofuels 2:11. https://doi.org/10.1186/1754-6834-2-11

12. Talebnia $F(2008)$ Ethanol production from cellulosic biomass by encapsulated Saccharomyces cerevisiae. Dissertation, Chalmers University of Technology

13. Ylitervo $P$ (2008) Production of ethanol and biomass from orange peel waste by Mucor indicus. Dissertation, University College of Borås

14. Grohmann K, Baldwin EA, Buslig BS (1994) Production of ethanol from enzymaticaily hydrolyzed orange peel by the yeast Saccharomyces cerevisiae. Appl Biochem Biotechnol 45(46):315-327

15. Zahi MR, El Hattab M, Liang H, Yuan Q (2017) Enhancing the antimicrobial activity of D-limonene nanoemulsion with the inclusion of $\varepsilon$-polylysine. Food Chem 221:18-23. https://doi. org/10.1016/j.foodchem.2016.10.037

16. Ambrosio CMS, Ikeda NY, Miano AC, Saldaña E, Moreno AM, Stashenko E, Contreras-Castillo CJ, Da Gloria EM (2019) Unraveling the selective antibacterial activity and chemical composition of citrus essential oils. Sci Rep 9:1-13. https://doi. org/10.1038/s41598-019-54084-3

Publisher's Note Springer Nature remains neutral with regard to jurisdictional claims in published maps and institutional affiliations. 